\title{
Photonic superdiffusive motion in resonance line radiation trapping Partial frequency redistribution effects
}

\author{
A. R. Alves-Pereira and E. J. Nunes-Pereira ${ }^{a)}$ \\ Escola de Ciências, Universidade do Minho, Centro de Física, 4710-057 Braga, Portugal \\ J. M. G. Martinho and M. N. Berberan-Santos \\ Centro de Química-Física Molecular, Instituto Superior Técnico, 1049-001 Lisboa, Portugal
}

(Received 17 January 2007; accepted 23 February 2007; published online 17 April 2007)

\begin{abstract}
The relation between the jump length probability distribution function and the spectral line profile in resonance atomic radiation trapping is considered for partial frequency redistribution (PFR) between absorbed and reemitted radiation. The single line opacity distribution function [M. N. Berberan-Santos et al., J. Chem. Phys. 125, 174308 (2006)] is generalized for PFR and used to discuss several possible redistribution mechanisms (pure Doppler broadening; combined natural and Doppler broadening; and combined Doppler, natural, and collisional broadening). It is shown that there are two coexisting scales with a different behavior: the small scale is controlled by the intricate PFR details while the large scale is essentially given by the atom rest frame redistribution asymptotic. The pure Doppler and combined natural, Doppler, and collisional broadening are characterized by both small- and large-scale superdiffusive Lévy flight behaviors while the combined natural and Doppler case has an anomalous small-scale behavior but a diffusive large-scale asymptotic. The common practice of assuming complete redistribution in core radiation and frequency coherence in the wings of the spectral distribution is incompatible with the breakdown of superdiffusion in combined natural and Doppler broadening conditions. (C) 2007 American Institute of Physics. [DOI: 10.1063/1.2717190]
\end{abstract}

\section{INTRODUCTION}

The study of transport phenomena, ${ }^{1}$ and in particular of diffusion processes, ${ }^{2}$ has developed considerably in the course of the past decades. One of the foundations of this area is the well-known diffusion equation, a consequence of the central limit theorem, ${ }^{3}$ that describes the random motion of a particle in an isotropic homogeneous three-dimensional space. The probability density function (PDF) of the diffusing species being at a certain position $r$ at time $t$, after a sufficiently high number of jumps and for an initial location at $r=0$, is given by the propagator ${ }^{4,5}$

$$
W(r, t) \propto \frac{1}{\left\langle r^{2}(t)\right\rangle^{3 / 2}} \exp \left(-\frac{3 r^{2} / 2}{\left\langle r^{2}(t)\right\rangle}\right),
$$

with the root-mean-square displacement,

$$
\left\langle r^{2}(t)\right\rangle=6 D t,
$$

where $D$ is the diffusion coefficient of the diffusing species.

By the end of the last century it was recognized that the simple behavior in Eqs. (1) and (2) was not completely general. The two most widely known deviations from the standard diffusive behavior are Lévy flights, ${ }^{6,7}$ coined and popularized by Benoit Mandelbrot, and Richardson's law of turbulent diffusion. ${ }^{8}$ Recently, anomalous diffusion equations were also found to apply in econometric modeling and/or prediction, ${ }^{9}$ in laser cooling of atoms, ${ }^{10}$ in the foraging be-

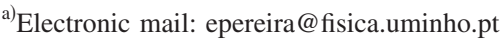

havior of some animals, ${ }^{11}$ and to the scaling laws ruling human travel activities (critically connected to the geographical spreading of infectious diseases). ${ }^{12}$

Situations deviating from the classical diffusion case can heuristically be described by a modified power law giving the scaling of the mean-squared displacement as

$$
\left\langle r^{2}(t)\right\rangle \propto t^{\gamma} .
$$

Anomalous diffusion results in the departure of the $\gamma$ factor from unity in the last equation, owing to the nonapplicability of the classical central limit theorem, as a result of the presence of broad distributions or long-range correlations and nonlocal effects. ${ }^{5,13}$ Broad spatial jump or waiting time distributions lead to a non-Gaussian and possibly a nonMarkovian time and spatial evolution of the system, ${ }^{4,5}$ giving rise to anomalous behaviors that can be collectively described as strange kinetics. ${ }^{14}$

Equation (3) encompasses both the linear dependence of the mean-squared displacement with time $(\gamma=1)$ characteristic of the standard Brownian motion as well as nonlinear dependencies, slower $(\gamma<1)$ and faster $(\gamma>1)$ than the classical case. The below Brownian motion regime is usually named subdiffusion or dispersive motion, while the above range is known as hyperdiffusion or superdiffusion. In order to generalize the classical treatment to consider anomalous diffusion, the continuous time random walk (CTRW) model is often considered. The random trajectory is viewed as the result of pairwise stochastically independent spatial and temporal increment events. When both the variance of the spatial steps and the expectation value of the temporal increments 
are finite, CTRW is equivalent to Brownian motion on large spatiotemporal scales ${ }^{5}$ and yields ordinary diffusion. Nevertheless, for the anomalous diffusion, a bifractional diffusion equation can be derived for the dynamics of $W(r, t)$. This can be solved using the methods of fractional calculus to give

$$
W(r, t) \underset{r, t \rightarrow \infty}{\sim} \frac{1}{t^{1 / \mu}} L_{\mu}\left(\frac{r}{t^{1 / \mu}}\right),
$$

where $L_{\mu}$ is a one-sided Lévy stable law of index $\mu$ (Ref. 13). This equation implies that the mean-squared displacement scales as

$$
p(r) \underset{r \rightarrow \infty}{\sim} r^{-(1+\mu)} .
$$

The particle's motion can be classified either as a Lévy flight, in the case that the time-of-flight of each jump in the trajectory is negligible, or as a Lévy walk, if the finite time to complete each jump must be taken into account. ${ }^{5,14}$

In this work we are interested in the stochastic theory of atomic resonance radiation migration under which the trajectories are of the superdiffusive type. The radiation transport is usually described in terms of a master equation, which has an integrodifferential form taking into account nonlocal effects. ${ }^{15}$ This is usually known as the Holstein-Biberman equation, named after seminal contributions in the 1940s. ${ }^{16-18}$ It was shown, first using ad hoc arguments ${ }^{19}$ and later demonstrated, that the motion of photons is superdiffusive. This was initially demonstrated for Doppler and Lorentz spectral line shapes and recently generalized for any line shape. ${ }^{15}$ The work was done in the limit of complete frequency redistribution (CFR), a situation in which the collision rate is sufficiently high when compared to the lifetime of the excited state to destroy any correlation between photon absorption and (re)emission events. This contribution aims to extend the previous work and discuss the influence of partial frequency redistribution (PFR) effects in the stochastic description of the resonance radiation trajectories. We will show that the overall asymptotic behavior of the trajectories is dictated by the redistribution in the rest frame of the atom, even if in the laboratory rest frame (LRF) memory effects persist on a finer scale. Specifically, we will show that the Doppler or Lorentz CFR superdiffusive asymptotics are recovered for PFR if there is only Doppler or Doppler plus natural redistribution in the atom frame. On the contrary, we show for the first time that the complete coherence in the atom's rest frame (ARF) will manifest itself as a breakdown of the superdiffusive behavior in all cases and not only for the wing frequency events.

In Sec. II the PFR effects are introduced along with the notation to be used. In Sec. III the single line opacity distribution function (ODF) under PFR is defined. This is subsequently used to derive the large-scale asymptotics of the jump length distribution in Sec. IV and of the opacity distribution in Sec. V. Section VI makes a connection between the superdiffusive character and the low opacity asymptotic behavior of the ODF. In Sec. VII numerical results are presented for the ODFs and the conditional jump length probability distribution functions, and the influence of frequency redistribution on both a small-scale (controlled by PFR ef- fects) as well as on a large-scale (corresponding to either a CFR superdiffusive or, in alternative, a diffusive) asymptotic is discussed. The main conclusions are summarized in Sec. VIII.

\section{PARTIAL FREQUENCY REDISTRIBUTION}

Resonance radiation trapping can be envisaged as a random flight with a jump size distribution dependent upon the spectral line shape. If all the scattering (absorption followed by reemission) events are coherent in frequency, the radiation transport is described by a diffusion equation with a diffusion coefficient dependent on the mean free path of the radiation. However, reabsorption-reemission events are inelastic, and a photon frequency redistribution in the laboratory reference frame exists preventing the notion of a mean free path. Therefore, the topology of the excitation random trajectory has to be related with the frequency redistribution of the emitted photons, since the jump length probability distribution function (PDF) must consider all possible optical emission frequencies. This can be done as ${ }^{19}$

$$
p(r)=\int_{-\infty}^{+\infty} \Theta(x) p(r \mid x) \mathrm{d} x=\int_{-\infty}^{+\infty} \Theta(x) \Phi(x) e^{-\Phi(x) r} \mathrm{~d} x,
$$

where $x$ is a frequency difference to the center-of-line frequency $x=\left(\nu-\nu_{0}\right) / W$, properly normalized by dividing by the corresponding width parameter [given for the Doppler and Lorentz spectral profiles as $W_{\text {Dop }}=v_{m p}\left(\nu_{0} / c\right)$ and $W_{\text {Lor }}$ $=\Gamma / 4 \pi$, respectively, where $v_{m p}$ is the most probable thermal velocity and $\Gamma$ is the radiative rate constant]; $\Theta(x)$ is the emission spectrum line shape; $p(r \mid x)$ is the photon exponential (Beer-Lambert) jump distribution; and $\Phi(x)$ is the normalized absorption line shape of the resonance line [so that $\left.\int_{-\infty}^{+\infty} \Phi(x) d x=1\right]$. The dimensionless distance $r=k_{0} / \Phi(0)$ defines an opacity or optical density scale from the center-ofline optical depth $k_{0}$ and the center-of-line absorption coefficient $\Phi(0)$. For a sufficiently high number of collisions during the lifetime of excited atoms, the emitted photon has a frequency completely uncorrelated with the absorption frequency and so the radiation is completely redistributed over the entire spectral line. This corresponds to the CFR case, for which the emission and absorption line shapes coincide and the jump length PDF is independent of the past history. For strong resonance lines with short lifetimes the frequency redistribution is partial since only a few collisions occur before emission, and consequently, the stochastic nature of the distribution must be considered. Partial frequency redistribution effects are important in an astrophysical context, as is the case of the scattering of Ly $\alpha$ radiation in optically thick nebulae $^{20}$ and the Ca I resonance line in the solar spectrum, ${ }^{21}$ as well as in laboratory scale atomic vapors, notably the 185-nm mercury line in low-pressure lighting discharges (which can account for as many as $10 \%$ of the overall flux in a typical T12 fluorescence lamp) ${ }^{22}$ and the 147- and 129-nm Xe VUV radiation used in plasma display panels (PDPs) applications. $^{23,24}$ For both mercury and xenon, the natural lifetimes of the corresponding excited states are less than about $3 \mathrm{~ns}$ and therefore only at very high densities is the 


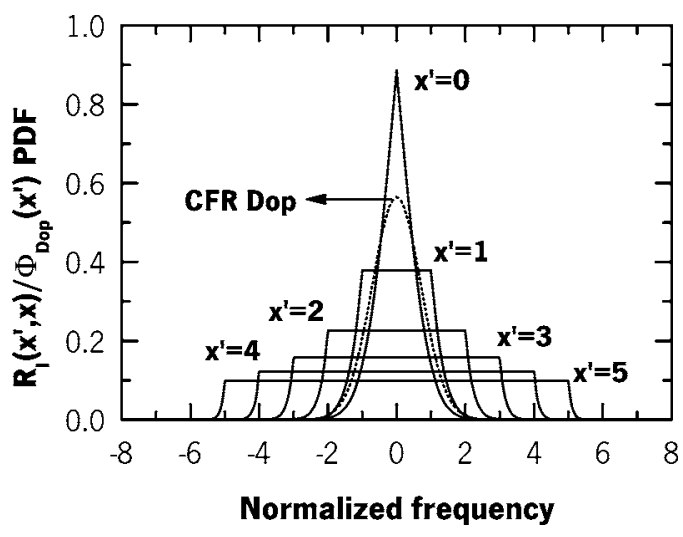

FIG. 1. Conditional reemission PDFs of partial redistribution function $R_{I}$ for several previous absorption frequencies $x^{\prime}$. The corresponding Doppler complete redistribution is also shown.

collision rate high enough to approach CFR conditions.

For a two-level model in the CFR limit, both absorption and emission spectra line shapes can be described by Doppler $\Phi_{\text {Dop }}(x)=(1 / \sqrt{\pi}) e^{-x^{2}}$, Lorentz $\Phi_{\text {Lor }}(x)=(1 / \pi) 1 /\left(1+x^{2}\right)$, or Voigt $\Phi_{\text {Voigt }}(x)=a / \pi^{3 / 2} \int_{-\infty}^{+\infty}\left[e^{-u^{2}} /\left(a^{2}+(x-u)^{2}\right)\right] \mathrm{d} u$ spectral distributions, in which $x$ is a normalized (with the Doppler width) difference to the center-of-line frequency and $a$ is the Voigt characteristic width, a ratio of the Lorentz to the Doppler widths defined previously. The study of PFR was pioneered in the 1940s in an astrophysical context and we will use the notation introduced by Hummer in the early 1960 s. $^{25}$ PFR is described by a redistribution $R$ function which is the joint probability of absorption of a photon with frequency $x^{\prime}$ and reemission of a photon with frequency $x$. This redistribution function is derived for a thermal vapor, assuming a Maxwell-Boltzmann velocity distribution for ground-state atoms and further imposing that the atoms' velocity is unchanged upon excitation (assumption not valid for ultracold vapors).

We will further consider unpolarized radiation (scalar redistribution function), use the angle averaged redistribution for an isotropic angular distribution reemission, and discuss the three most important cases for a ground-state absorbing state: (i) pure Doppler broadening $\left(R_{I}\right)$, (ii) combined natural and Doppler broadening $\left(R_{I I}\right)$, and (iii) combined Doppler, natural, and collisional broadening $\left(R_{I I I}\right)$ (Refs. 16, 20, 26, and 27). For the $R_{I}$ case, both levels are infinitely sharp and there is pure coherent scattering in the atom's rest frame. This is unrealistic because both the lower and upper levels of the optical transition are broadened to some extent by radiation damping and/or collisions. It is nevertheless useful since it allows the separation of the Doppler shift from the other broadening mechanisms. The physical picture for $R_{I I}$ is a line with an infinitely sharp lower level and an upper level broadened only by radiation damping. In the ARF the absorption is Lorentzian and scattering is completely (frequency) coherent. This type of scattering is important for resonance lines in low-density media where the collisions do not perturb the excited levels. $R_{I I I}$ applies whenever the upper state is broadened by radiation and collision damping in the limit where collisions are frequent enough to cause complete frequency redistribution in the ARF. Both absorption and reemission profiles are Lorentzian in the atom's frame with a total width equal to the sum of the radiative plus collisional (uncorrelated) characteristic widths. It is well known that complete redistribution in the ARF does not imply the same quantitative behavior in the laboratory frame used to describe radiation transport. Under the previously mentioned assumptions, the laboratory frame joint redistribution functions are given by $^{26,28}$

$$
\begin{aligned}
R_{I}\left(x^{\prime} ; x\right)= & \frac{1}{2} \operatorname{erfc}(\bar{x}), \\
R_{I I}\left(x^{\prime} ; x\right)= & \frac{1}{\pi^{3 / 2}} \int_{\frac{1}{2}\left|x-x^{\prime}\right|}^{+\infty} e^{-u^{2}}\left\{\arctan \left[\frac{x+u}{a}\right]\right. \\
& \left.-\arctan \left[\frac{\bar{x}-u}{a}\right]\right\} \mathrm{d} u,
\end{aligned}
$$

and

$$
\begin{aligned}
R_{I I I}\left(x^{\prime} ; x\right)= & \frac{1}{\pi^{5 / 2}} \int_{0}^{+\infty} e^{-u^{2}}\left\{\arctan \left[\frac{x^{\prime}+u}{a}\right]\right. \\
& \left.-\arctan \left[\frac{x^{\prime}-u}{a}\right]\right\}\left\{\arctan \left[\frac{x+u}{a}\right]\right. \\
& \left.-\arctan \left[\frac{x-u}{a}\right]\right\} \mathrm{d} u,
\end{aligned}
$$

where $a$ is the Voigt width, erfc is the complementary error function, and $\underline{x}$ and $\bar{x}$ stand for $\underline{x} \equiv \min \left(\left|x^{\prime}\right|,|x|\right)$ and $\bar{x}$ $\equiv \max \left(\left|x^{\prime}\right|,|x|\right)$.

In Eq. (6) we need to use the (re)emission PDF. For radiation transport in thermal vapors we can assume a CFR absorption profile which corresponds to the ensemble average over the Maxwellian velocity distribution. The reemission distribution comes from the joint redistribution probability and, according to Bayes rule, is given by

$$
\Theta\left(x \mid x^{\prime}\right)=\frac{R\left(x^{\prime} ; x\right)}{\Phi\left(x^{\prime}\right)},
$$

and it is this conditional (re)emission PDF that should be used. $\Phi\left(x^{\prime}\right)$ is the absorption spectrum PDF which should be either the pure Doppler distribution $\left(R_{I}\right)$ or the Voigt profile $\left(R_{I I}\right.$ and $\left.R_{I I I}\right)$.

Figures 1-3 show the spectral distributions calculated from the previous equations. Both $R_{I}$ and $R_{I I I}$ distributions are symmetric, while $R_{I I}$ is asymmetric. In all cases, a complete redistribution in the ARF does not imply CFR in the laboratory frame; the degree of redistribution in the laboratory frame being always higher for core radiation and for the $R_{I}$ and $R_{I I I}$ cases. The complete coherence in the atom's rest frame for the $R_{I I}$ model is not complete in the laboratory frame. However, the coherence degree is more important for wing photons and higher $a$ values (bigger importance of natural coherent broadening compared to Doppler). On the other hand, for the $R_{I I I}$ distribution, the increase of the $a$ value implies higher redistribution in the core (bigger importance of collisional redistributed broadening). We will discuss now the influence of the redistribution between absorp- 

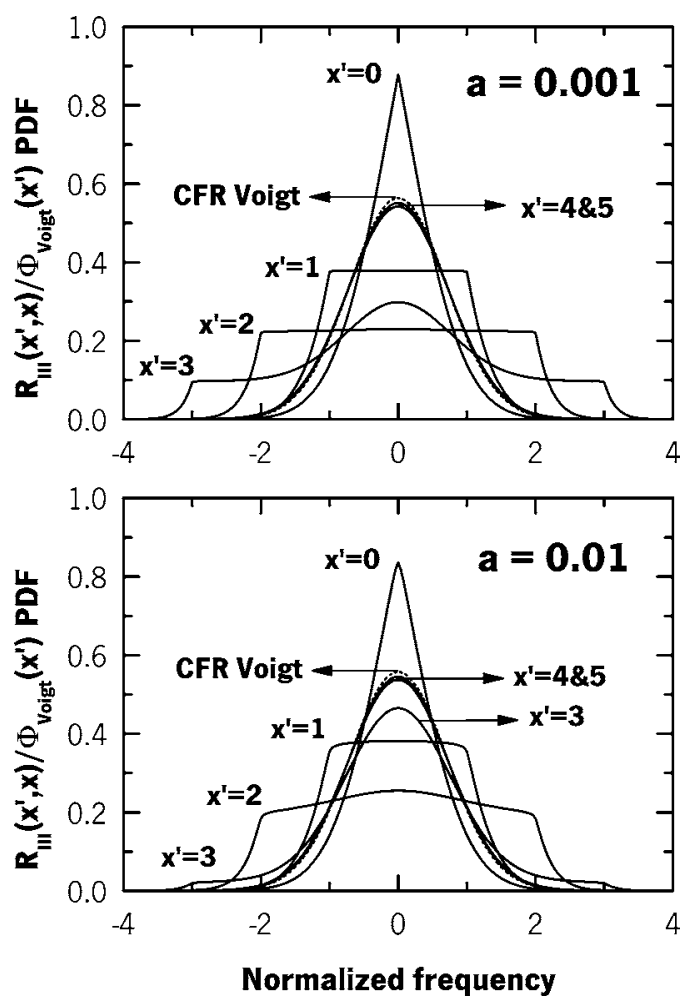

FIG. 2. Conditional reemission PDFs of partial redistribution function $R_{I I I}$ for several previous absorption frequencies $x^{\prime}$ and Voigt width parameters $a$. The corresponding Voigt complete redistribution is also shown.

tion and reemission in the asymptotic superdiffusive character of photon migration under PFR conditions. For this purpose we extend the concept of a single line opacity distribution function to the case of incomplete redistribution.

\section{LINE OPACITY DISTRIBUTION FUNCTION UNDER PARTIAL REDISTRIBUTION}

To study the superdiffusive behavior of resonance radiation trapping it is convenient to discuss the influence of the line shape on the opacity (probability) distribution function. For this we will use a line opacity scale, defined from the normalized absorption line shape as $k \equiv \Phi(x)$. This line opacity scale will thus have no reference to the actual system size or number density and will be useful only for infinite media. This is the one chosen to discuss the ODF. The monochromatic line opacity along a given path length $l$, for homogeneously distributed particles, is $k(x)=n \sigma_{0} l \Phi(x) / \Phi(0)$ $=k_{0} \Phi(x) / \Phi(0)=\Phi(x) r$ and is therefore proportional to the number density $n$ (or pressure) and to the center-of-line opacity $k_{0}=n \sigma_{0} l$, where $\sigma_{0}$ is the center-of-line absorption cross section as usual. The discussion of the spectral line profile influence on the jump length distribution will use an overall (for the whole line) dimensionless opacity or optical density scale defined as $r=k_{0} / \Phi(0)$ so that $r=\int_{-\infty}^{+\infty} k(x) \mathrm{d} x$. This is done since any given jump distance must reflect the whole of the line shape and not just a given monochromatic opacity.

We will now generalize the basic procedure used previously ${ }^{15}$ for the complete frequency distribution to cover the partial frequency distribution case. We will start with the
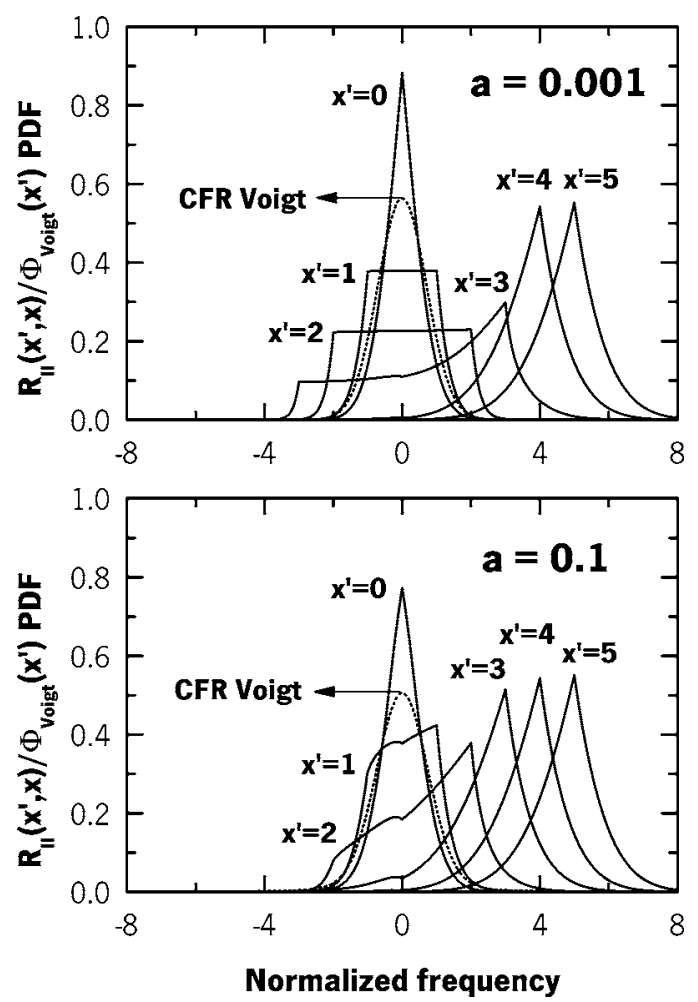

FIG. 3. Conditional reemission PDFs of partial redistribution function $R_{I I}$ for several previous absorption frequencies $x^{\prime}$ and Voigt width parameters $a$. The corresponding Voigt complete redistribution is also shown.

jump length PDF by rewriting Eq. (6), making explicit the conditional dependence in the absorption frequency,

$$
p\left(r \mid x^{\prime}\right)=\int_{-\infty}^{+\infty} \Theta\left(x \mid x^{\prime}\right) \Phi(x) \mathrm{e}^{-\Phi(x) r} \mathrm{~d} x .
$$

Since photon migration by reabsorption is associated with exponential jump size PDFs, it is desirable to consider the general $p\left(r \mid x^{\prime}\right)$ as a linear combination of exponential densities given by

$$
p\left(r \mid x^{\prime}\right)=\int_{0}^{+\infty} H\left(k \mid x^{\prime}\right)\left(k e^{-k r}\right) \mathrm{d} k,
$$

where $H\left(k \mid x^{\prime}\right)$ is the PDF of effective line opacities. The single line opacity at a given frequency, $x$, is given by $k(x)$ $\equiv \Phi(x)$. As was shown before for the CFR case, $k H\left(k \mid x^{\prime}\right)$ is the inverse Laplace transform of $p\left(r \mid x^{\prime}\right)$. The inversion is analytical and can be performed using the real inversion form of the Laplace transform (with the parameter $c=0$ ) (Ref. 29),

$$
\begin{aligned}
k H\left(k \mid x^{\prime}\right)= & \frac{1}{\pi} \int_{0}^{+\infty}\{\operatorname{Re}[p(i \omega)] \cos (k \omega) \\
& -\operatorname{Im}[p(i \omega)] \sin (k \omega)\} \mathrm{d} \omega,
\end{aligned}
$$

which gives ${ }^{15}$ 


$$
H\left(k \mid x^{\prime}\right)=\frac{1}{k} \int_{-\infty}^{+\infty} \Theta\left(x \mid x^{\prime}\right) \Phi(x) \delta[k-\Phi(x)] \mathrm{d} x .
$$

This can be further simplified by making the change of variable $y=\Phi(x)$ and decomposing the integration into positive and negative $x$ frequencies,

$$
H\left(k \mid x^{\prime}\right)=-\frac{1}{k} \int_{0}^{\Phi(0)}\left[\theta_{+}(k)+\theta_{-}(k)\right] y \Psi^{\prime}(y) \delta[k-y] \mathrm{d} y,
$$

since it is assumed that $\Phi(x)$ is always non-negative, and that the transformation has opposite signs for positive and negative frequencies. $\Psi(y)$ is the inverse function of $\Phi(x)$, $\Psi(y)=\Phi^{-1}(y)$. The integration in $y$ only runs to the maximum value of the absorption spectrum [the center-of-line absorption coefficient, $\Phi(0)]$ and $\theta_{+}(k)$ and $\theta_{-}(k)$ represent the value of the (conditional) probabilities for emission for positive and negative frequencies, implicitly given from the opacity $k$ through the absorption spectrum. For any particular opacity $k$, there are two (symmetrical) frequencies, $+x$ and $-x$, that give rise to $k$ defined by $\Phi(x)=k$ (symmetric absorption). These frequencies do not necessarily have the same reemission probability. One can therefore define $\theta_{+}(k)$ $=\Theta\left(x \mid x^{\prime}\right)$ and $\theta_{-}(k)=\Theta\left(-x \mid x^{\prime}\right)$ and finally rewrite Eq. (15) as

$$
H\left(k \mid x^{\prime}\right)=\left\{\begin{array}{cl}
-\left[\theta_{+}(k)+\theta_{-}(k)\right] \Psi^{\prime}(k) & \text { if } k<\Phi(0) \\
0 & \text { if } k \geq \Phi(0)
\end{array} .\right.
$$

This is the general workhorse that will be used in the following sections to discuss the influence of PFR in resonance radiation reabsorption and compare it with the CFR case. ${ }^{15}$ We will show how the redistribution of the emission into the wings of the absorption spectrum implies a superdiffusive behavior characterized by a very broad (infinite mean) jump size distribution. In addition, we are able to demonstrate that, if redistribution into the tails of the absorption is prevented due to PFR memory effects, the superdiffusive character breaks down, and this can be seen by the appearance of a cutoff in the PDF of the opacity at small line opacity values, which is translated into a corresponding cutoff of the higher jump lengths.

Two of the PFR cases defined before $\left(R_{I}\right.$ and $\left.R_{I I I}\right)$ have symmetric reemission PDFs (see Figs. 1 and 2), which allow us to write

$$
H\left(k \mid x^{\prime}\right)=\left\{\begin{array}{cl}
-2 \theta_{+}(k) \Psi^{\prime}(k) & \text { if } k<\Phi(0) \\
0 & \text { if } k \geq \Phi(0)
\end{array} .\right.
$$

For complete frequency redistribution this gives

$$
H\left(k \mid x^{\prime}\right)=\left\{\begin{array}{cl}
-2 k \Psi^{\prime}(k) & \text { if } \quad k<\Phi(0) \\
0 & \text { if } k \geq \Phi(0)
\end{array},\right.
$$

which is simply the result obtained before. ${ }^{15}$

\section{ASYMPTOTIC BEHAVIOR OF THE JUMP DISTRIBUTION}

Using Eq. (16), Eq. (12) can be rewritten as

$$
p\left(r \mid x^{\prime}\right)=-\int_{0}^{\Phi(0)}\left[\theta_{+}(k)+\theta_{-}(k)\right] \Psi^{\prime}(k)\left(k e^{-k r}\right) \mathrm{d} k .
$$

The asymptotic behavior is easily found by performing the change of variable $y=k r$,

$$
p\left(r \mid x^{\prime}\right)=-\frac{1}{r^{2}} \int_{0}^{\Phi(0) r}\left[\theta_{+}\left(\frac{y}{r}\right)+\theta_{-}\left(\frac{y}{r}\right)\right] \Psi^{\prime}\left(\frac{y}{r}\right)\left(y e^{-y}\right) \mathrm{d} y,
$$

which gives

$$
p\left(r \mid x^{\prime}\right) \underset{r \rightarrow \infty}{\sim} r^{-2} \int_{0}^{+\infty}\left[\theta_{+}\left(\frac{y}{r}\right)+\theta_{-}\left(\frac{y}{r}\right)\right] \Psi^{\prime}\left(\frac{y}{r}\right)\left(y e^{-y}\right) \mathrm{d} y .
$$

For PFR conditions, we have to consider both the absorption as well as the conditional reemission asymptotics. For the absorption spectrum, if the line shape function can be given by a power-law asymptotic in the wings, ${ }^{15,19}$ then one has

$$
k(x) \equiv \Phi(x) \underset{x \rightarrow \infty}{\sim} x^{-p_{a}} \text { with }\left(p_{a}>1\right),
$$

where $a$ stands for absorption.

The inverse function is

$$
\Psi(k) \underset{k \rightarrow 0}{\sim} k^{-1 / p_{a}},
$$

which can be written as

$$
\Psi^{\prime}\left(\frac{y}{r}\right)_{r \rightarrow \infty}^{\sim}-\frac{r}{p_{a} y} y^{-1 / p_{a}} r^{1 / p_{a}} .
$$

If the asymptotic of the conditional reemission is similar to that of absorption (but possibly with a different parameter value), then

$$
\Theta\left(x \mid x^{\prime}\right) \underset{r \rightarrow \infty}{\sim} x^{-p_{e}} \text { with }\left(p_{e}>1\right),
$$

where $e$ stands for emission. Therefore, both $\theta_{+}$and $\theta_{-}$scale as

$$
\theta_{+}(k) \sim \theta_{-}(k) \underset{k \rightarrow 0}{\sim} k^{p^{\prime} e^{\prime p}} .
$$

Finally, Eq. (21) can be rewritten as

$$
\begin{aligned}
p\left(r \mid x^{\prime}\right) \underset{r \rightarrow \infty}{\sim} r^{-2} \int_{0}^{+\infty}\left(\frac{y}{r}\right)^{p_{e} / p_{a}}\left[-\left(\frac{r}{p_{a} y}\right) y^{\left.-1 / p_{a} r^{1 / p_{a}}\right]}\left(y e^{-y}\right) \mathrm{d} y\right. \\
=\Gamma\left(1+\frac{p_{e}}{p_{a}}-\frac{1}{p_{a}}\right) r^{1 / p_{a}-\left(1+p_{e} / p_{a}\right)},
\end{aligned}
$$

and therefore

$$
p\left(r \mid x^{\prime}\right) \underset{r \rightarrow \infty}{\sim} r^{1 / p_{a}-\left(1+p_{e} / p_{a}\right)} .
$$

Comparing the previous equation with the Lévy flight asymptotic in Eq. (5) gives 


$$
\mu=\frac{1}{p_{a}}\left(p_{e}-1\right) .
$$

If the asymptotic regime is the same for both absorption and PFR reemission, $p=p_{a}=p_{e}$ and the jump size distribution has the asymptotic

$$
p\left(r \mid x^{\prime}\right) \underset{r \rightarrow \infty}{\sim} r^{-(2-1 / p)},
$$

which corresponds to the CFR form obtained before. ${ }^{15}$ So, the CFR asymptotic is recovered even in PFR conditions, as long as the asymptotic forms of both the absorption spectrum and the conditional reemission are the same. The Lévy parameter is also the one obtained in CFR conditions,

$$
\mu=1-\frac{1}{p} \text {. }
$$

\section{ASYMPTOTIC BEHAVIOR OF THE OPACITY DISTRIBUTION}

We can obtain the same conclusions from the spectral and from the ODF PDFs asymptotics. Indeed, the small opacity scaling law can be easily obtained from the previous equations. From Eqs. (22) and (23), one obtains

$$
\Psi^{\prime}(k) \underset{k \rightarrow 0}{\sim}-\frac{1}{p_{a}} k^{-\left(1 / p_{a}+1\right)} .
$$

Using the reemission asymptotic of Eq. (26), the ODF small opacity limit is

$$
H\left(k \mid x^{\prime}\right) \underset{k \rightarrow 0}{\sim}-\frac{1}{p_{a}} k^{-\left[1 / p_{a}+\left(1-p_{e} / p_{a}\right)\right]},
$$

which, for absorption and reemission spectral distributions with the same asymptotic character $p=p_{a}=p_{e}$, gives

$$
H\left(k \mid x^{\prime}\right) \underset{k \rightarrow 0}{\sim}-\frac{1}{p} k^{-1 / p} .
$$

The last two equations show that the ODF asymptotic behavior can be easily identified from a log-log plot as

$$
\log H\left(k \mid x^{\prime}\right) \underset{k \rightarrow 0}{\sim}-\left[\frac{1}{p_{a}}+\left(1-\frac{p_{e}}{p_{a}}\right)\right] \log k
$$

and

$$
\log H\left(k \mid x^{\prime}\right) \underset{k \rightarrow 0}{\sim}-\frac{1}{p} \log k .
$$

The cases of CFR Doppler and Lorentz resonance line radiation transfer give analytical ODF expressions and were considered in previous works. ${ }^{15,19}$ For the Doppler case, one has $\mu \simeq 1$ and thus Eq. (22) is only approximately valid and the dependence of the ODF over the opacity values is extremely weak. However, for Lorentz, the asymptotic in Eq. (22) is exact with $p=2$. The ODF is given by ${ }^{15}$

$$
H_{\text {Lor }}(k)=\frac{\Phi(0)}{k}\left(\frac{\Phi(0)}{k}-1\right)^{-1 / 2},
$$

which has an exact asymptotic of the form given by Eq. (36).

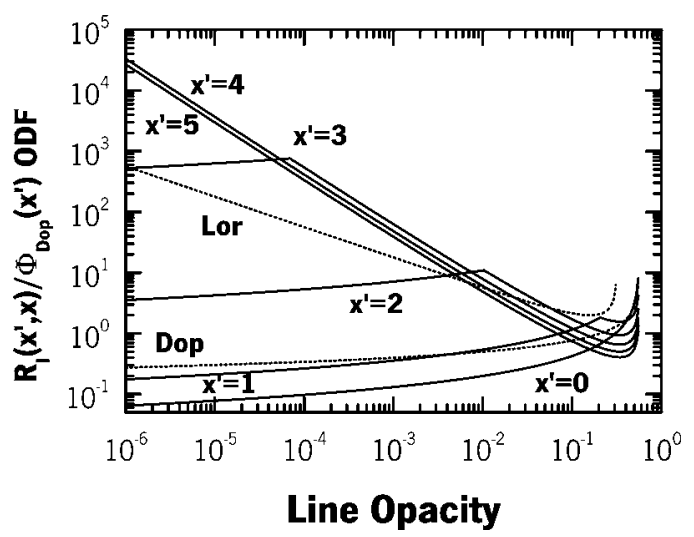

FIG. 4. Conditional ODF for partial redistribution function $R_{I}$ for several previous absorption frequencies $x^{\prime}$. The complete redistribution limiting Doppler and Lorentz cases are also shown.

\section{SUPERDIFFUSIVE BEHAVIOR FOR RESONANCE LINE TRAPPING}

Although the superdiffusive behavior is well established from the asymptotics derived in the previous two sections, a simpler more informative, alternative approach is possible. Equation (12) can be written in Laplace space as

$$
\hat{p}\left(u \mid x^{\prime}\right)=\int_{0}^{\Phi(0)} \frac{k H\left(k \mid x^{\prime}\right)}{u+k} \mathrm{~d} k,
$$

and since the moments of the jump size distribution can be recast from the $m$ th derivatives of this Laplace transform as $\left.\hat{p}^{(m)}\left(u \mid x^{\prime}\right)\right|_{u=0}=(-1)^{m}\left\langle r^{m}\right\rangle$, one obtains

$$
\left\langle r^{m}\right\rangle=m ! \int_{0}^{\Phi(0)} \frac{H\left(k \mid x^{\prime}\right)}{k^{m}} \mathrm{~d} k=m !\left\langle k^{-m}\right\rangle .
$$

The integration only goes up to the upper limit of the center-of-line opacity. The first moment gives $\langle r\rangle=\int_{0}^{\Phi(0)}\left[H\left(k \mid x^{\prime}\right) / k\right] d k$ which shows that, unless $H\left(k \mid x^{\prime}\right)$ vanishes as $k \rightarrow 0$, an infinite mean jump size is obtained.

If one considers now the asymptotic of Eq. (34), one can conclude that $\langle r\rangle \propto \lim _{k \rightarrow 0^{+}}\left(1 / k^{1 / p}\right)$, which is finite only if $1 / p \leq 0$. But, since $p \geq 1$ by construction, one can finally conclude that the mean jump size is infinite irrespective of the actual value of the $p$ parameter.

\section{NUMERICAL RESULTS}

Figures 4-6 give the conditional ODFs for the three PFR redistribution mechanisms considered in this work alongside with the corresponding CFR Doppler and Lorentz limiting cases. The results were computed from Eqs. (16) and (17). The CFR ODFs allow one to quantify very easily the cause of the superdiffusive behavior for resonance line radiation trapping; for very small opacities, the ODF either decreases very slowly (Doppler) or even increases in importance (Lorentz) with a decrease in opacity values. It does not vanish in the limit of $k \rightarrow 0$ and therefore superdiffusion sets in (see previous section). From Eq. (12), one can see that the slower the decrease in the ODF for smaller opacity values, the more important the higher jump sizes. For Doppler and Lorentz (and Voigt) CFR trapping, the ODF variation for small $k$ 
values is either increasing or so slowly decreasing as to render even the first moment of the jump size distribution infinite. ${ }^{15,19}$ The CFR Voigt case is a self-affine multifractal with two different characteristic Lévy flight $\mu$ 's parameters, a $\mu \simeq 1$ corresponding to the Doppler-like core radiation, and a $\mu=1 / 2$ for Lorentz-like wing photons. The overall excitation random walk topology is controlled by the bigger Lorentz scale (if not truncated by the vapor confining boundaries). ${ }^{19}$ Therefore, in the present case of PFR effects in an infinite medium, and even for the Voigt Maxwellian absorption profile, we need only concern ourselves with the PFR deviation from the most extreme Lorentz CFR asymptotic.

Figure 4 shows that the superdiffusive character of $R_{I}$ partial redistribuion is similar to the one for the corresponding Doppler CFR case. There is a transition between two regimes: (i) the central core constant redistribution (compare Fig. 1), characterized by a faster than Lorentz increase of opacity PDF for small opacity values, and (ii) the wings' Doppler-like redistribution, with a Doppler-like (albeit with a slower convergence) asymptotic. The transition between the two regimes depends upon the actual value of the previous absorption frequency $x^{\prime}$ (for $x^{\prime}=4$ and 5, the transition still occurs but at opacity values smaller than the ones represented in Fig. 4).

The $R_{I}$ case is useful because it allows one to single out the pure Doppler effect on the redistribution functions. The more realistic cases are described by the $R_{I I}$ or $R_{I I I}$ distributions. Figure 5 shows the conditional ODFs for the $R_{I I I}$ redistribution for representative values of the $a$ parameter found in experimental conditions. The PFR random walk has a superdiffusive character with the same asymptotic of the CFR Lorentz. For the $R_{I I I}$ combined Doppler, natural, and collisional broadening, there is complete (Lorentzian) redistribution in the atom's rest frame, but not in the laboratory frame. One can distinguish mainly two regimes, one for very small line opacities and the other for opacities approaching the center-of-line value, the exact transition between the two depending upon the $a$ parameter. For very small line opacities, the actual superdiffusive regime corresponds to the CFR Lorentz case. For higher line opacities, the redistribution is similar to $R_{I}$, an initial step increase in ODF PDF with decreasing opacity, followed by a Doppler-like asymptotic (which eventually merges into the Lorentz higher scale asymptotic $^{15}$ ). The $a$ parameter quantifies the relative importance of Lorentz (natural plus collisional in this case) over Doppler; the higher the $a$ value the more important the Lorentz complete redistribution in the atom's rest frame and therefore the smaller the deviations from CFR and the onset of the CFR Lorentz asymptotic occurs earlier (i.e., for higher opacity values).

The conditional ODFs for the $R_{I I}$ redistribution are shown in Fig. 6. The most remarkable difference from the previous cases resides in the low line opacity asymptotic. There is a very abrupt cutoff of the ODF PDF values for small opacities which scales approximately as $a$. This case corresponds to excited levels not perturbed by collisions during their lifetime and coherent reemission in the ARF. This coherent reemission in the ARF does not give automatically
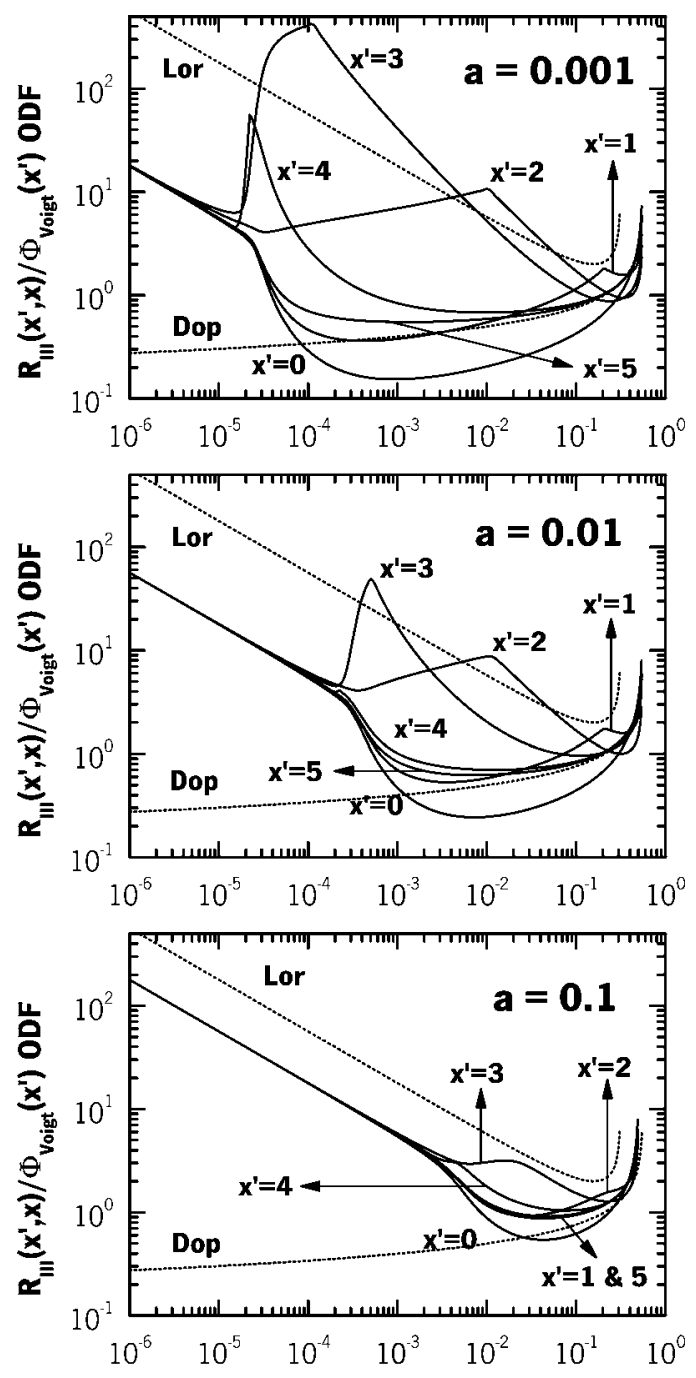

Line Opacity

FIG. 5. Conditional ODF for partial redistribution function $R_{I I I}$ for several previous absorption frequencies $x^{\prime}$ and Voigt width parameters $a$. The complete redistribution limiting Doppler and Lorentz cases are also shown.

coherence in the LRF due to the Maxwell velocity distribution. However, after several Doppler widths (depending on $a$ ), the ARF coherency appears in the cutoff of the ODF. As for the previous $R_{I I I}$ case, two regimes can be distinguished with a transition between them that depends on $a$. For line opacities approaching the center-of-line value, $\Phi(0)$, the redistribution is similar to the pure Doppler $R_{I}$ case; an initial very steep (faster than Lorentzian and persisting to smaller line opacities the more off center the previous photon absorption is) increase with decreasing opacity value followed by a CFR Doppler redistribution until the small opacities approaching the cutoff values (compare the CFR asymptotic with the ODF curves for the $x^{\prime}=0,1$, and 2 cases). The cutoff of the ODF PDF for the smaller line opacities is very important since it shows the breakdown of the superdiffusion character for this $R_{I I}$ model.

From the above results some general conclusions can be extracted: (i) the ARF frequency coherence does not immediately give rise to LRF coherence due to the ensemble Maxwellian velocity distribution averaging and (ii) the ARF re- 

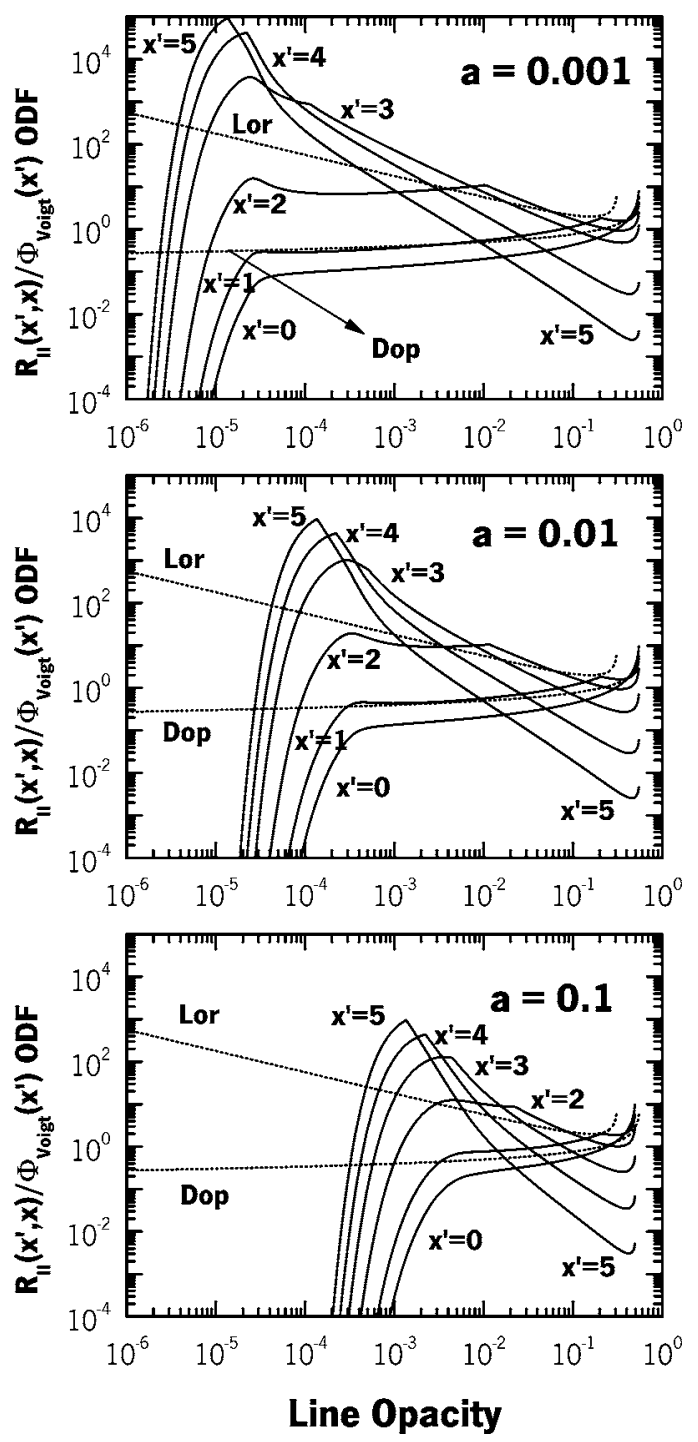

FIG. 6. Conditional ODF for partial redistribution function $R_{I I}$ for several previous absorption frequencies $x^{\prime}$ and Voigt width parameters $a$. The complete redistribution limiting Doppler and Lorentz cases are also shown.

distribution (or nonredistribution) character will eventually show up as a corresponding LRF asymptotic for sufficiently small line opacities (that is to say, for optically thick enough vapors). The CFR in the $\mathrm{ARF}$ for the $R_{I I I}$ will give rise to a Lorentz CFR (superdiffusive) asymptotic in infinite media even under partial redistribution while the complete coherence in the ARF for $R_{I I}$ will reveal itself in the LRF as a breakdown of the superdiffusive character. The breakdown of the superdiffusion for thick vapors under $R_{I I}$ conditions is particularly important due to the widespread practice of approximating the actual complex behavior of the $R_{I I}$ redistribution by a linear superposition of complete frequency redistribution in the line core plus a complete coherence in the line wings. This practice is essentially motivated by the mathematical simplification that it allows and it can be traced back to the work of Jefferies and White ${ }^{30}$ (and is therefore known as the JW approximation). Although the several implementations of the JW approximation differentiate the photons from the core from those from the wings differently, all ignore the possibility that even the most extreme wing

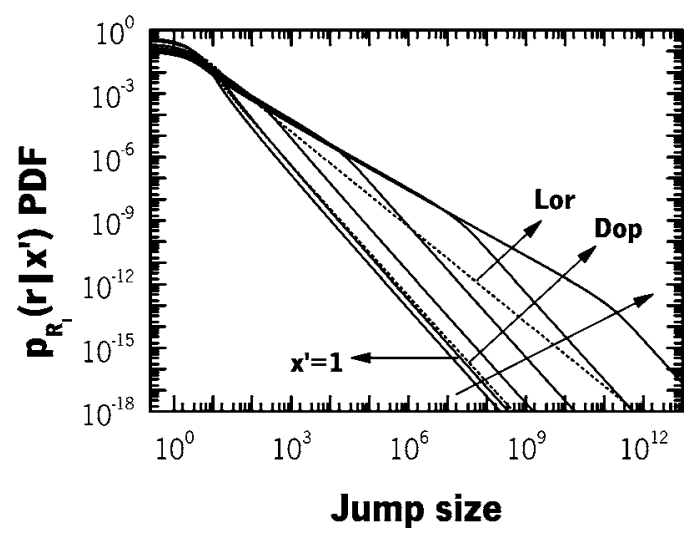

FIG. 7. Conditional one-sided jump PDF for partial redistribution function $R_{I}$ for several previous absorption frequencies $x^{\prime}$ in the sequence $x^{\prime}=0,1,2$, 3,4 , and 5 (arrow direction). The complete redistribution limiting Doppler and Lorentz cases are also shown.

photons can be redistributed in frequency, thus neglecting the so-called "diffusion" in frequency. Moreover, the full redistribution function is skewed toward the line center and this is not taken into account. Figure 6 clearly shows another physical limitation of the JW approximation, which is generally not pointed out. For high opacity samples, the CFR assumption in core radiation is a severe approximation since CFR implies that radiation well into the wings can be emitted in a single scattering event, which cannot occur (an upper limit exists) due to the breakdown of the Doppler asymptotic. This contributes to show that JW-type approximations are only acceptable for small Voigt parameters and for overall centerof-line opacities roughly smaller than 100-500 (Refs. 16, 24, and 31).

The asymptotic analysis of resonance radiation trapping started probably with Holstein's seminal work ${ }^{18}$ and received afterwards a relevant contribution from van Trigt's infinite opacity expansion analysis in the 1970 s. ${ }^{32}$ Asymptotic analysis is a very useful tool to calculate the large-scale behavior, which, from the derived scaling laws, can provide physical insight into more complex and realistic conditions. This is particularly true for partial redistribution in the LRF. We clearly show that the large-scale behavior is of the CFR Lorentz superdiffusive type for complete redistribution in the ARF but of the diffusive character if the excited atom is intrinsically coherent (even if the small scale resembles the pure Doppler superdiffusive character). So one can use a generalized diffusive transport equation ${ }^{5}$ for line radiation in this case, even if the effective mean transport coefficients will hide the details of the small-scale partial redistribution effects. The present work is, in this respect, complementary of the asymptotic analysis of the integral equation of line radiation transfer carried out by Frisch. ${ }^{20,33}$ The work presented here is much simpler and straightforward than Frisch's approach. Furthermore, we clearly identify the CFR in the line core JW-type approximation for $R_{I I}$ as incompatible with the large-scale diffusive behavior.

Figures 7-9 give the jump size PDFs computed numerically from Eq. (11). These give essentially the same information as the ODF PDFs, although in terms of the overall opacity length which is directly related to physical distances (for 
an additional note on the relation of opacity scales and physical distances, see Ref. 15). The superdiffusive behavior of $R_{I}$ and $R_{I I I}$ is evident, while the breakdown of superdiffusion is manifested by the abrupt cutoff for $R_{I I}$ at higher jump lengths. Smaller details can also be pointed out. For $R_{I}$, there is an initial higher than superdiffusive Lorentz jump PDF which changes into the large-scale Doppler asymptotic at high distances (transition not shown but confirmed for the last two cases of $x^{\prime}=4$ and 5 in Fig. 7). For the $R_{I I I}$ redistribution, all cases converge into the same Lorentzian CFR. And finally, for the $R_{I I}$ case there are three characteristic jump length ranges: a first in which the jump PDF increases faster for decreasing opacities than CFR Lorentz, an intermediate with a CFR Doppler character (and these two distance ranges roughly correspond to the pure Doppler $R_{I}$ case), and a third one corresponding to the diffusive onset due to a step cutoff for higher distances.

The jump size PDFs define the overall opacity scale in which partial frequency redistribution effects are important as well as the transition from superdiffusion like into standard diffusion for the $R_{I I}$ case. From Fig. 8 one expects that the onset of CFR Lorentz asymptotic for high opacities should roughly scale with the width $a$ parameter; PFR effects should manifest themselves at distances up to $10^{5}, 10^{4}$, and $10^{3}$ for $a=0.001,0.01$, and 0.1 , respectively, when measured in a dimensionless overall opacity scale. On the other hand, in the case of $R_{I I}$ redistribution, Fig. 9 shows that the standard diffusion should become noticeable only at very high opacities; roughly $10^{6}, 10^{5}$, and $10^{4}$ for $a=0.001,0.01$, and 0.1 , respectively. At smaller scales, and notably for finite systems with the biggest dimension not exceeding these opacity values, the faster than standard diffusion behavior is dominant.

\section{DISCUSSION AND CONCLUSIONS}

The line opacity distribution function approach was used to discuss the superdiffusive character of resonance line radiation transfer under partial frequency redistributions effects, as well as the conditions for its breakdown. These effects are the result of the ensemble average from the atom's rest frame redistribution function into the laboratory frame (used to describe radiation transport). We discuss PFR effects for three frequency redistribution functions between absorption and reemission: $R_{I}$ (pure Doppler broadening), $R_{I I}$ (Doppler plus natural), and $R_{I I I}$ (combined Doppler, natural, and collisional broadening). The Doppler ARF of $R_{I}$ will give rise to the complete frequency redistribution superdiffusive Doppler asymptotic for sufficiently small line opacities, while the Lorentz ARF intrinsics of $R_{I I I}$ give also small line opacity large-scale Lorentzian CFR superdiffusive asymptotics. However, the ARF frequency coherence of $R_{I I}$ gives rise to a different behavior, because the abrupt cutoff in the jump size distribution for higher distances means that there is a breakdown of the superdiffusive behavior. This is not recognized in both the astrophysical spectral line formation as well as in the laboratory scale atomic vapors studies. This breakdown means that the popular JW-type approximations of PFR under $R_{I I}$ conditions are a severe approximation, since
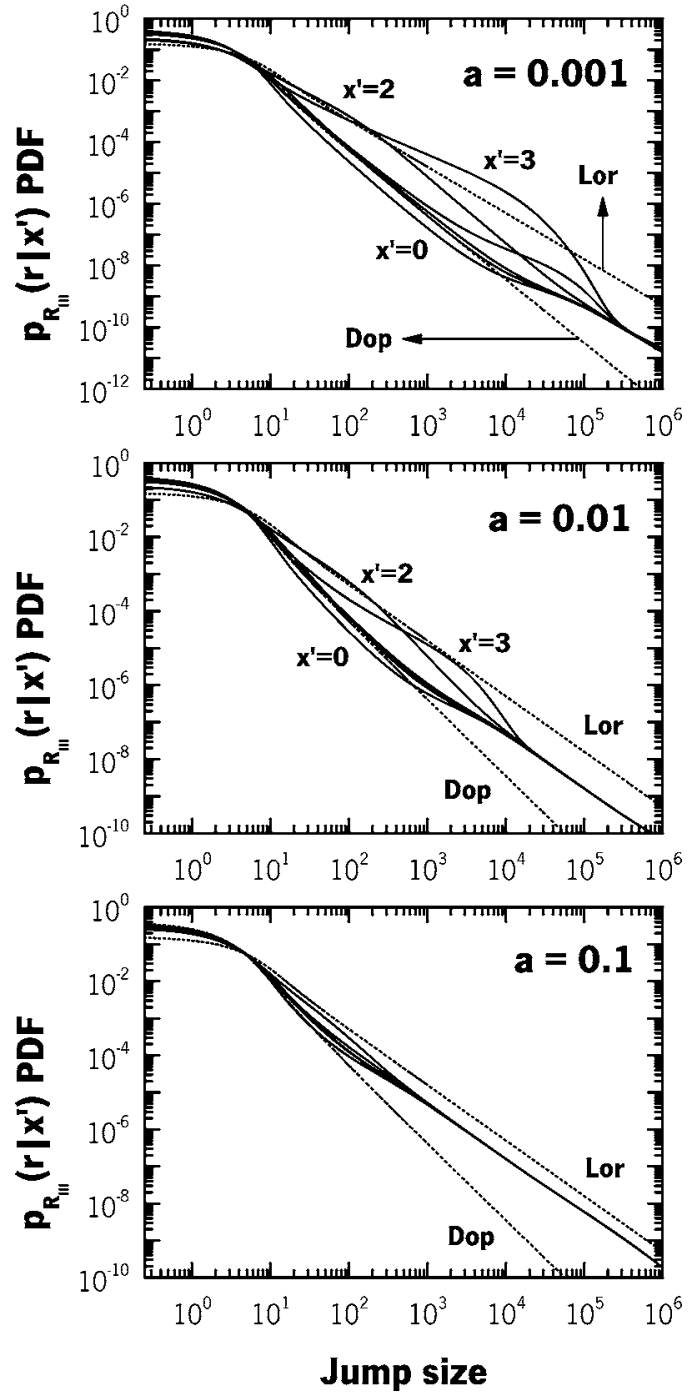

FIG. 8. Conditional one-sided jump PDF for partial redistribution function $R_{I I I}$ for several previous absorption frequencies $\left(x^{\prime}=0,1,2,3,4\right.$, and 5) and Voigt width parameters $a$. The complete redistribution limiting Doppler and Lorentz cases are also shown.

they assume that core radiation CFR allows a single scattering to photons well into the absorption wings. Under experimental conditions rendering $R_{I I}$ realistic, a generalized diffusion equation should be valid even for core line radiation. This has a classical analog found in physical kinetics, where the macroscopic hydrodynamic behavior is derived from a microscopic description through a Chapman-Enskog expansion. ${ }^{1}$

The $R_{I I}$ and $R_{I I I}$ redistribution functions assume either a complete frequency coherence or a complete redistribution in the ARF, respectively. These situations should correspond to two limiting cases for which the collisions during the excited state lifetime are almost nonexistent or very frequent. For resonance lines, the general form of the redistribution function should be $\mathrm{b}^{31,33,34}$

$$
R\left(x^{\prime} ; x\right)=\left(1-P_{c}\right) R_{I I}\left(x^{\prime} ; x\right)+P_{c} R_{I I I}\left(x^{\prime} ; x\right),
$$

where the branching ratio $P_{c}=\Gamma_{c} /\left(\Gamma_{c}+\Gamma_{\text {rad }}\right)$ is defined as the probability that an elastic collision will destroy the correlation between the absorbed and reemitted frequencies in the 

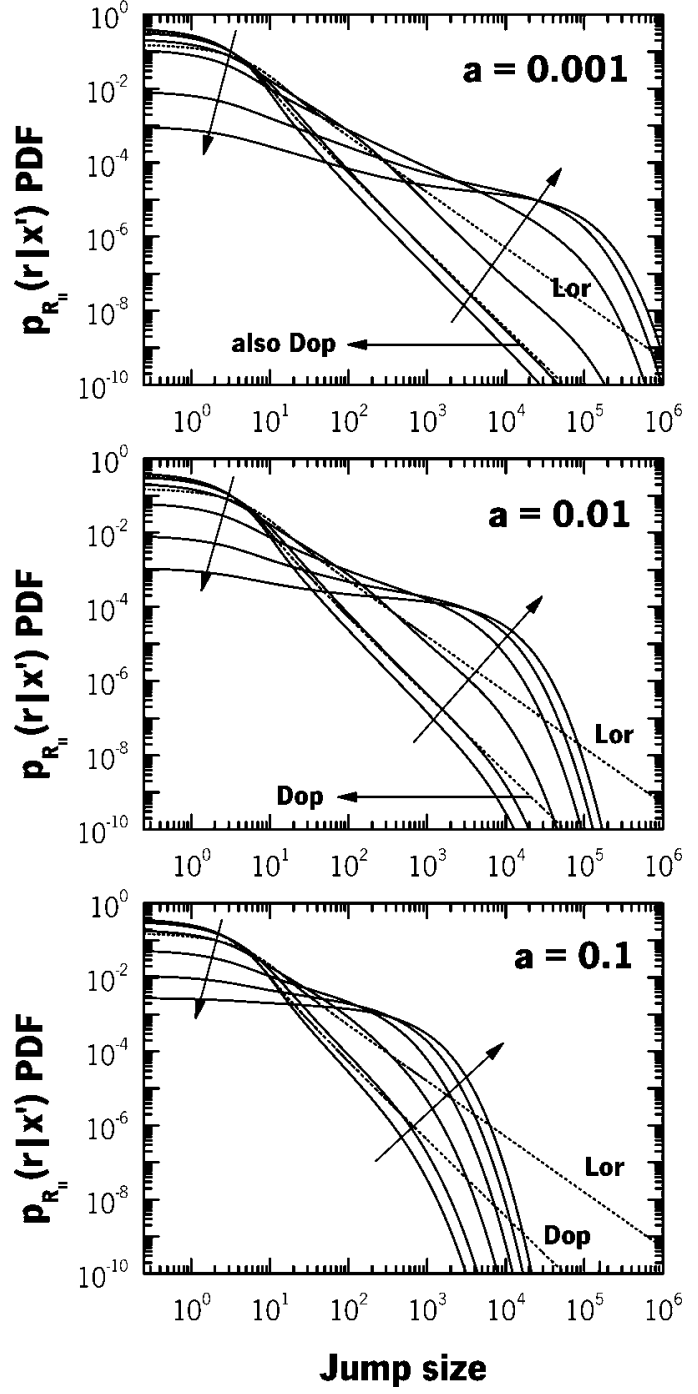

FIG. 9. Conditional one-sided jump PDF for partial redistribution function $R_{I I}$ for several previous absorption frequencies $x^{\prime}$ and Voigt width parameters $a$. The arrow direction shown results in the sequence $x^{\prime}=0,1,2,3,4$, and 5. The complete redistribution limiting Doppler and Lorentz cases are also shown.

ARF, and should go to one in the limit of high densities and to zero in the low-density limit. This equation reflects the competition between complete redistribution in the ARF on the one hand and almost complete coherence on the other hand, depending on the elastic collisional rate. Presumably, below some critical value for $P_{c}, R_{I I}$-type redistribution will dictate a large-scale diffusive behavior while after that critical value, $R_{I I I}$ will take control of the large-scale behavior giving rise to superdiffusion. The transition condensed in Eq. (40) is important since the classical landmark work of Post in the 1980s for trapping under PFR with 185-nm $\mathrm{Hg}$ radiation $^{31}$ corresponds to a range of $P_{c}$ values roughly from $1 \%$ to $30 \%$.

Figures 4-9 display the conditional ODFs and jump size distributions for a given previous absorption optical frequency. The radiation migration in physical space is not a Markov process whenever PFR is meaningful since the whole hierarchy of the process cannot be constructed from the spatial distribution of the excitation in a given initial time plus the single (spatial) transition probability (contrary to the CFR case where the spatial transition probability is independent of past history). However, the spatial evolution of excitation can be embedded in a Markov process defined as the excitation migration in both spatial and frequency space. ${ }^{4}$ This extended Markov process will consider explicitly the joint redistribution between absorption and reemission frequencies, information that otherwise would be contained implicitly in the past values of the absorption frequencies. In principle, it would even be possible to define two different Markov processes for all the $R$ 's redistribution mechanisms, depending on the coarsening level of the description. ${ }^{4}$ On a fine level description, one should have a complete stochastic formulation of the radiation trapping problem encompassing both the small-scale (controlled by PFR) as well as the largescale (asymptotic) behaviors. But one could also devise a coarser level description retaining only the large-scale asymptotic and blurring the small-scale PFR details into a transition probability valid only for large distances. For the $R_{I}$ and $R_{I I I}$ cases, the large-scale transition probability would be given by the corresponding Doppler or Lorentz CFR superdiffusive asymptotic while for the $R_{I I}$ a simpler diffusiontype behavior should apply.

Finally we discuss the applicability of the opacity distribution function formalism to astrophysical applications. In astrophysics, we have to deal with a very large number of spectral lines that overlap. Typically, consideration all overlapping spectral lines (plus continua) in reactionhydrodynamic modelling is prohibitive, but this can be circumvented by the use of an ODF. Detailed studies of this approach have shown that ODFs reproduce satisfactorily both emergent fluxes and the physical stellar atmospheric structure. ${ }^{26}$ In astrophysical radiative transfer problems, each individual opacity will be the result of grouping several lines. Recently, Wehrse and co-workers have used a Poisson distributed point process for the statistical description of the number of lines. ${ }^{35}$ However, they did not attempt a comprehensive investigation of the dependencies of the ODFs on the detailed line profiles. The present work is a contribution toward the improvement of this approach by including the effect of the absorption and reemission profiles in a similar way used by us to study the anomalous diffusion resulting from the redistribution along a single line profile.

\section{ACKNOWLEDGMENTS}

This work was supported by Fundação para a Ciência e Tecnologia (FCT, Portugal) within Project No. POCI/QUI/ 58535/2004 and by FCT and Universidade do Minho (Portugal) within Project No. REEQ/433/EEI/2005. It also used computational facilities bought under Project No. POCTI/ CTM/41574/2001, funded by FCT and the European Community Fund FEDER. A.R.A.-P. also acknowledges FCT funding under Reference No. SFRH/BD/4727/2001.

\footnotetext{
${ }^{1}$ S. Chapman and T. G. Cowling, The Mathematical Theory of Nonuniform Gases, 3rd ed. (Cambridge University Press, Cambridge, England, 1970). ${ }^{2}$ R. M. Mazo, Brownian Motion, Fluctuations, Dynamics and Applications (Oxford University Press, Oxford, England, 2002); B. D. Hughes, Random Walks and Random Environments (Oxford University Press, Oxford, England, 1995), Vol. 1.
} 
${ }^{3}$ B. V. Gnedenko and A. N. Kolmogorov, Limit Distributions for Sums of Independent Random Variables (Addison-Wesley, Reading, 1954).

${ }^{4}$ N. G. van Kampen, Stochastic Processes in Physics and Chemistry (North-Holland, Amsterdam, 1992).

${ }^{5}$ R. Metzler and J. Klafter, Phys. Rep. 339, 1 (2000); J. Phys. A 37, R161 (2004).

${ }^{6}$ B. B. Mandelbrot, The Fractal Geometry of Nature (Freeman, New York, 1983).

${ }^{7}$ Lévy Flights and Related Topics in Physics, edited by M. F. Shlesinger, G. M. Zaslavsky, and U. Frisch (Springer, Berlin, 1995).

${ }^{8}$ M. F. Shlesinger, B. J. West, and J. Klafter, Phys. Rev. Lett. 58, 1100 (1987).

${ }^{9}$ J. P. Bouchaud and M. Potters, Theory of Financial Risk and Derivative Pricing: From Statistical Physics to Risk Management (Cambridge University Press, Cambridge, England, 2000).

${ }^{10}$ F. Bardou, J. P. Bouchaud, A. Aspect, and C. Cohen-Tannoudji, Lévy Statistics and Laser Cooling (Cambridge University Press, Cambridge, England, 2002).

${ }^{11}$ G. M. Viswanathan, V. Afanasyev, S. V. Buldyrev, E. J. Murphy, P. A. Prince, and H. E. Stanley, Nature 381, 413 (1996).

${ }^{12}$ D. Brockmann, L. Hufnagel, and T. Geisel, Nature 439, 462 (2006).

${ }^{13}$ J. P. Bouchaud and A. Georges, Phys. Rep. 195, 127 (1990).

${ }^{14}$ M. F. Shlesinger, G. M. Zaslavsky, and J. Klafter, Nature 363, 31 (1993).

${ }^{15}$ M. N. Berberan-Santos, E. J. Nunes-Pereira, and J. M. G. Martinho, J. Chem. Phys. 125, 174308 (2006).

${ }^{16}$ A. F. Molisch and B. P. Oehry, Radiation Trapping in Atomic Vapours (Oxford University Press, Oxford, England, 1998).

${ }^{17}$ M. N. Berberan-Santos, E. Pereira, and J. M. G. Martinho, in Resonance Energy Transfer, edited by D. L. Andrews and A. A. Demidov (Wiley, Chichester, 1999), p. 108.

${ }^{18}$ T. Holstein, Phys. Rev. 72, 1212 (1947).
${ }^{19}$ E. Pereira, J. M. G. Martinho, and M. N. Berberan-Santos, Phys. Rev. Lett. 93, 120201 (2004).

${ }^{20}$ H. Frisch, Astron. Astrophys. 87, 357 (1980).

${ }^{21}$ H. Frisch, Sol. Phys. 164, 49 (1996).

${ }^{22}$ G. G. Lister, J. E. Lawler, W. P. Lapatovich, and V. Godyak, Rev. Mod. Phys. 76, 541 (2004)

${ }^{23}$ S. Rauf and M. J. Kushner, J. Appl. Phys. 85, 3460 (1999); 85, 3470 (1999); T. van der Straaten and M. J. Kushner, ibid. 87, 2700 (2000).

${ }^{24}$ H. M. Anderson, S. D. Bergeson, D. A. Doughty, and J. E. Lawler, Phys. Rev. A 51, 211 (1995).

${ }^{25}$ D. G. Hummer, Mon. Not. R. Astron. Soc. 125, 21 (1962).

${ }^{26}$ D. Mihalas, Stellar Atmospheres, 2nd ed. (Freeman, San Francisco, 1978).

${ }^{27}$ I. Hubeny, in Progress in Stellar Spectral Line Formation Theory, edited by J. E. Beckman and L. Crivellari (Reidel, Dordrecht, 1985), p. 27.

${ }^{28}$ C. J. Cannon, The Transfer of Spectral Line Radiation (Cambridge University Press, Cambridge, England, 1985).

${ }^{29}$ M. N. Berberan-Santos, J. Math. Chem. 38, 165 (2005).

${ }^{30}$ J. T. Jefferies and O. R. White, Astrophys. J. 132, 767 (1960); J. T. Jefferies, Spectral Line Formation (Blaisdell, Waltham, 1968).

${ }^{31}$ H. A. Post, Phys. Rev. A 33, 2003 (1986); H. A. Post, P. van de Weijer, and R. M. M. Cremers, ibid. 33, 2017 (1986).

${ }^{32}$ C. van Trigt, Phys. Rev. 181, 97 (1969); Phys. Rev. A 1, 1298 (1970); 4, 1303 (1971); 13, 726 (1976); 13, 734 (1976).

${ }^{33}$ H. Frish, in Progress in Stellar Spectral Line Formation Theory, edited by J. E. Beckman and L. Crivellari (Reidel, Dordrecht, 1985), p. 87.

${ }^{34}$ A. Omont, E. W. Smith, and J. Cooper, Astrophys. J. 175, 185 (1972).

${ }^{35}$ B. Baschek, W. von Waldenfels, and R. Wehrse, Astron. Astrophys. 371, 1084 (2001); R. Wehrse, B. Baschek, and W. von Waldenfels, ibid. 390, 1141 (2002) 